\title{
Coxsackievirus A16 in a 1-Day-Old Mouse Model of Central Nervous System Infection Shows Lower Neurovirulence than Enterovirus A71
}

\author{
Y. T. Hooi ${ }^{*}$, K. C. Ong ${ }^{\dagger}$, S. H. Tan ${ }^{\dagger}$, D. Perera ${ }^{\dagger}$ and K. T. Wong* \\ * Department of Pathology, ${ }^{\dagger}$ Department of Biomedical Science, Faculty of Medicine, University of Malaya, Kuala Lumpur \\ and ${ }^{\ddagger}$ Institute of Health and Community Medicine, Universiti Malaysia Sarawak, Sarawak, Malaysia
}

\begin{abstract}
Summary
Coxsackievirus A16 (CV-A16) and enterovirus A71 (EV-A71) are the major causes of hand, foot and mouth disease in young children. Although less so with CV-A16, both viruses are associated with serious neurological syndromes, but the differences between their central nervous system infections remain unclear. We conducted a comparative infection study using clinically-isolated CV-A16 and EV-A71 strains in a 1-day-old mouse model to better understand the neuropathology and neurovirulence of the viruses. New serotype-specific probes for in situ hybridization were developed and validated to detect GV-A16 and EV-A71 RNA in infected tissues. Demonstration of $\mathrm{CV}-\mathrm{A} 16$ virus antigens/RNA, mainly in the brainstem and spinal cord neurons, confirmed neurovirulence, but showed lower densities than in EV-A71 infected animals. A higher lethal dose ${ }_{50}$ for $\mathrm{CV}$ A16 suggested that CV-A16 is less neurovirulent. Focal virus antigens/RNA in the anterior horn white matter and adjacent efferent motor nerves suggested that neuroinvasion is possibly via retrograde axonal transport in peripheral motor nerves.
\end{abstract}

(c) 2020 Elsevier Ltd. All rights reserved.

Keywords: coxsackievirus A16; enterovirus A71; mouse model; neurovirulence

\section{Introduction}

Coxsackievirus A16 (CV-A16) and enterovirus A71 (EV-A71), both serotypes in the same species A group in the genus Enterovirus, family Picornaviridae, are the most common enteroviruses that cause hand, foot and mouth disease (HFMD) (Repass et al., 2014). The viral RNA genomes are approximately 7.4 kilobases and each contain a single open reading frame flanked by a $5^{\prime}$ non-translated region (NTR) and $3^{\prime}$ NTR. The genome encodes four viral structural proteins (VP4, VP2, VP3 and VP1) and seven nonstructural proteins $(2 \mathrm{~A}, 2 \mathrm{~B}, 2 \mathrm{C}, 3 \mathrm{~A}, 3 \mathrm{~B}, 3 \mathrm{C}$ and $3 \mathrm{D})$ (Solomon et al., 2010).
HFMD usually presents in young children with mild clinical manifestations, including fever, blisters or rashes on the hands and feet, and ulcers or sores on the buccal mucosa and tongue (Liu et al., 2011b). Both EV-A71 and CV-A16 are recognized as causes of HFMD outbreaks in many countries over recent years (Koh et al., 2016); however, animal model studies and vaccine development have been more focused on EV-A71 due to the higher prevalence of severe or even fatal central nervous system (CNS) complications. GV-A16-associated HFMD was generally thought to be mild and self-limiting (Chang et al., 1999; Mao et al., 2014); however, there are increasing reports that $\mathrm{GV}-\mathrm{A} 16$ can also cause severe neurological complications (e.g. aseptic meningitis, rhombencephalitis, lethal myocarditis and pneumonitis), albeit far less commonly than 